\title{
Innovative approach to the medication verification process: Role of pharmacists in the minimization of confirmation bias
}

\author{
Tu T. Tran*1, Bryan Pham ${ }^{2}$, Tiana Vo ${ }^{1}$, Jane Hodding ${ }^{1}$, Jennifer Le ${ }^{2}$ \\ ${ }^{1}$ Department of Inpatient Pharmacy, Long Beach Memorial Hospital, Long Beach CA, USA \\ ${ }^{2}$ Skaggs School of Pharmacy and Pharmaceutical Sciences, University of California San Diego, La Jolla CA, USA
}

Received: February 5, 2020

Accepted: May 22, 2020

Online Published: June 15, 2020

DOI: $10.5430 /$ ijh.v6n2p62

URL: https://doi.org/10.5430/ijh.v6n2p62

\begin{abstract}
Purpose: Medication errors may stem from confirmation bias, a type of selective thinking to support one's own preconception rather than what is actually true. Our objective was to describe an innovative approach to the medication verification process that minimizes confirmation bias.

Summary: In September 2014, our pharmacy team implemented a unique double-check method that limited confirmation bias within the medication verification process for the compounding of non-sterile oral products and sterile intravenous admixtures. Our innovative approach required the pharmacist to first check the constituents and the final product without directly viewing the label, and then double check against the final product label. A confirmation bias form was used to ensure adherence to the newly designed workflow. The primary factor contributing to confirmation bias was expectation. Over the 29-month study period, we observed an immediate reduction in escaped defects (30 pre- to 0-4 post-implementation). Most of the escaped defects were "potential" medications errors that were captured prior to leaving the pharmacy and did not reach the patient nor resulted in any harm. While this process improved medication safety, there was no change in pharmacy staff requirement, indicating its cost neutrality.

Conclusion: The elimination of confirmation bias and incorporation of a double-check system during the drug verification process improved the safe use of medications without additional staffing cost.
\end{abstract}

Key Words: Verification, Errors, Confirmation, Bias

\section{INTRODUCTION}

Medication errors are an important public health concern since they can lead to significant adverse drug reactions that result in hospitalization and even death. ${ }^{[1,2]}$ Approximately $5 \%$ of medication orders contain an error, in which half of these orders reach patients. ${ }^{[3-5]}$ Alarmingly, 180,000 patient die each year from an adverse event and more than $1,000,000$ adverse drug events that occur in the hospital are preventable. ${ }^{[6]}$

The Institute of Medicine's “To Err is Human" emphasizes the need to prevent medication errors through system improvement. ${ }^{[7]}$ As such, developing and implementing processes to safeguard the public against medication errors is essential to maximizing patient safety. ${ }^{[5,8,9]}$ One critical step in preventing medication errors occurs before dispensing the drug and involves pharmacist verification of the drug to the

*Correspondence: Tu T. Tran; Email: ttran11@memorialcare.org; Address: 2801 Atlantic Ave, PO Box 1428, Long Beach, CA 90801-1428, USA. 
original prescription. ${ }^{[10]}$ Confirmation bias may occur during this key step of the pharmacy's medication use process that may potentially lead to medications errors and consequently, adverse drug events.

In an effort to ensure the safe and effective use of medications, the inpatient pharmacists at MemorialCare Long Beach Medical Center (LBMC) and MemorialCare Miller Children's and Women's Hospital of Long Beach (MCWHLB) established a unique method of drug verification (or checking). This report describes the development and implementation of this innovative approach to the drug verification process that minimizes confirmation bias.

\section{MEDICATION VERIFICATION PROCESS}

There are key steps within the systematic medication use process: prescribing, verification, dispensing, administering, and monitoring. A medication error can occur at any one of these steps. Medication errors resulting from the verification and dispensing steps are specific to pharmacy and can be mitigated by system improvement. According to the Institute for Safe Medication Practices, there are two stages of the pharmacy verification process: verification I occurs before product preparation when the pharmacist checks the prescription for appropriateness of the drug, dose, allergy, and any other clinical aspect of the physician's order; and verification II ensures the accuracy of product preparation and labeling before it is dispensed for patient use. ${ }^{[5]}$ For this article, we focused on system improvement in the verification II process to mitigate the risk of developing medication errors.

The verification II step of the medication use process involves various intricacies that may lead to a medication error leaving the pharmacy and potentially affecting the patient. The role of a pharmacist in an inpatient setting is to provide medications to our patients, who are acutely ill, in the safest and most time-efficient way. Certain operational factors may contribute to the occurrence of a medication error, including workload, work environment, time of day, type of shift, and number of working pharmacists or technicians. In particular, an increase in the number of drug orders verified has been associated with an increase in the rate medication errors during verification. ${ }^{[11]}$ This potentiates the risk of a medication errors due to the certain operational factors as described above. As such, system strategies to minimize these operational factors should be evaluated and implemented to mitigate the risk of medication use in patients.

\section{Confirmation bias}

As a widely applicable concept, confirmation bias may be present in many different settings and contribute to an outcome. In healthcare, the Institute for Safe Medication Prac- tices issued an alert stating that some medication errors occur due to confirmation bias, which is an inattentional blindness that affects humans. ${ }^{[11]}$ Confirmation bias is a type of selective thinking in which there exists a tendency to interpret or recall information to support one's own preconception, rather than what is actually true. As Mark Twain commented "It ain't what you know what gets you in trouble, it's what you know for sure that ain't so." As a form of cognitive bias that is involuntary and unnoticed by the executor, some examples of confirmation bias related to medication errors, which includes mixing up drug names that sound similar, mixing up drug strengths, and assuming the concentration of an IV medication is correct because it is a routine medication.

The four factors that captures human attention and hence may contribute to confirmation bias are expectation, conspicuity, mental workload and task interference, and capacity. ${ }^{[11]}$ Expectation affects our ability to pay attention, especially to information that is consistent with one's own belief. Conspicuity describes how the physical properties of information, such as a high degree of contrast with the background or the perceived relevance of the information, can attract someone's attention. Multi-tasking or low workload may lead to inattentional blindness as both reduce mental attention given to the designated tasks. The capacity to pay attention varies between individuals and is influenced by various factors, such as age and fatigue.

Confirmation bias may influence the medication verification process when the pharmacist checks a medication dispensed against the original order. Unfortunately, medication errors may ensue when confirmation bias is present, even within a vigilant and meticulous system of pharmacy verification. As such, minimizing confirmation bias can be one of the ways within the pharmacy verification process to avert medication errors.

\section{ORgANizATIONAL STRUCTURE}

Within the integrated Memorial Care Health System are two hospitals, namely LBMC and MCWHLB. LBMC is a notfor-profit, community-based, tertiary care, teaching hospitals with a level 2 trauma center and 497 (61 intensive care) and 348 (137 intensive care) beds, respectively. Based on the four factors that contribute to confirmation bias, examples of medication errors that have occurred during the drug verification process at these hospitals are described in Table 1.

Traditionally, the pharmacists prepare and check medications in a very systematic fashion. For example, non-sterile compounding of oral drug dilution utilizes a recipe card for reconstitution or preparation instruction is used. Strictly adhering to the direction, the pharmacist examines the recipe 
card and then checks the final product that is prepared by a pharmacy technician against the recipe card. As such, an inherent bias may theoretically have been introduced into this process since the pharmacist already reviewed the recipe card prior to verifying the final product. Prior knowledge that provides an idea of what to expect may elicit a false sense of affirmation, affecting an individual's perception.

The verification process for the sterile compounding of in- travenous (IV) medications is similar to that of oral preparations. ${ }^{[12]}$ The pharmacist examines the drug label and then compares it to the drug vials and diluents used to prepare the IV admixture. Confirmation bias inherently may arise when the pharmacist presumably checks the IV preparation with the preconceived notion of the concordance between the drug label and the drug vial. This prior knowledge of what to expect again may elicit a false sense of affirmation that can contribute to a potential medication error.

Table 1. Medication errors during the medication verification process

\begin{tabular}{|c|c|c|}
\hline Medication Error & Healthcare Personnel & $\begin{array}{l}\text { Factor Contributing } \\
\text { to Confirmation Bias }\end{array}$ \\
\hline $\begin{array}{l}\text { Incorrect drug selected for mini-bag cartfill, resulting in ceftriaxone } 1 \mathrm{~g} \\
\text { instead of ceftriaxone } 2 \mathrm{~g} \text { per order. }\end{array}$ & Pharmacy Technician & Expectation \\
\hline $\begin{array}{l}\text { Incorrect diluent selected to make pantoprazole } 80 \mathrm{mg} \text { in D5W } 200 \mathrm{ml} \text {, } \\
\text { rather than correctly using normal saline. }\end{array}$ & Pharmacy Technician & Expectation \\
\hline $\begin{array}{l}\text { Two product labels printed and both were pulled and filled by the } \\
\text { pharmacist concurrently. One order had piperacillin/tazobactam } 3.375 \mathrm{~g} \\
\text { in normal saline } 100 \mathrm{ml} \text { and the other, for midazolam } 100 \mathrm{mg} \text { in D5W } \\
100 \mathrm{ml} \text {. Pharmacist pulled both drugs at the same time, incorrectly affixed } \\
\text { piperacillin/tazobactam label on midazolam bag and vice versa. Patient } \\
\text { received } 100 \mathrm{mg} \text { of midazolam administered over } 30 \text { minutes and } \\
\text { experienced adverse events that required flumazenil administration. }\end{array}$ & Pharmacist & $\begin{array}{l}\text { Mental workload and } \\
\text { task interference } \\
\text { Capacity, as product } \\
\text { preparation occurred in } \\
\text { the early morning at the } \\
\text { near end of a night shift }\end{array}$ \\
\hline Incorrect volume of diluent to make vancomycin $83 \mathrm{mg} / \mathrm{ml}$ oral solution. & $\begin{array}{l}\text { Pharmacy Technician and } \\
\text { Pharmacist }\end{array}$ & Expectation \\
\hline
\end{tabular}

\section{INNOVATIVE APPROACH TO MEDICATION VERIFICATION PROCESS}

Confirmation bias introduced during our traditional medication verification process for oral and IV compounding medications contributed to medication errors. The traditional model of checking both oral/IV compounded medications as well as unit dose medications involved checking the label against what is being dispensed by the technician. This inherently introduces confirmation bias because the checker expects to see what is on the label and is more inclined to believe what is pulled is the correct strength/form of the medication needed. Using our traditional standardized method of verification and dispensing, approximately 30 "escaped defects" (or system catches) per month were identified prior to September 2014 (see Figure 1). Notably, the primary factor contributing to this confirmation bias largely stems from expectation. ${ }^{[11]}$ Some examples of escaped defects included incorrect concentration of an oral dilution, wrong drug for the IV product, and incorrect diluent for a docked Baxter mini-bag system.

Most of the escaped defects were "potential" medications errors that were fortunately captured prior to leaving the pharmacy and subsequently did not reach the patient nor resulted in any harm. Most system catches were identified by the pharmacists during the verification process when the pharmacists check for the correct diluent volume (termed volume check) and active ingredient prior to compounding by the technician. Other defects were caught at the "final check" when the pharmacist ensures that all ingredients were mixed correctly. Catching a defect at this last step is very time-consuming and sometimes costly (depending on the product) since the technician must re-make the product from beginning.

All escaped defects that occurs are recorded and categorized as A or B based on the National Coordinating Council for Medication Error Reporting and Prevention (NCCMERP). ${ }^{[13]}$ The NCC-MERP index for categorizing Medication Errors ranges from Categories A through Categories I. Categories A and B were considered potential errors that were identified early in the medication use process and subsequently did not result in any medication error nor reached the patient. Categories $\mathrm{C}$ and $\mathrm{D}$ were considered potential errors that reached the patient and caused no harm. Categories $\mathrm{E}$ through $\mathrm{H}$ are considered potential medication errors that reach the patient and caused some harm. Categories I are considered potential medication errors that reached the pa- 
tient and resulted in patient death. In a proactive effort to mize confirmation bias within our drug verification process. minimize these system catches that may inadvertently hinder patient safety, we developed an innovative approach to mini-

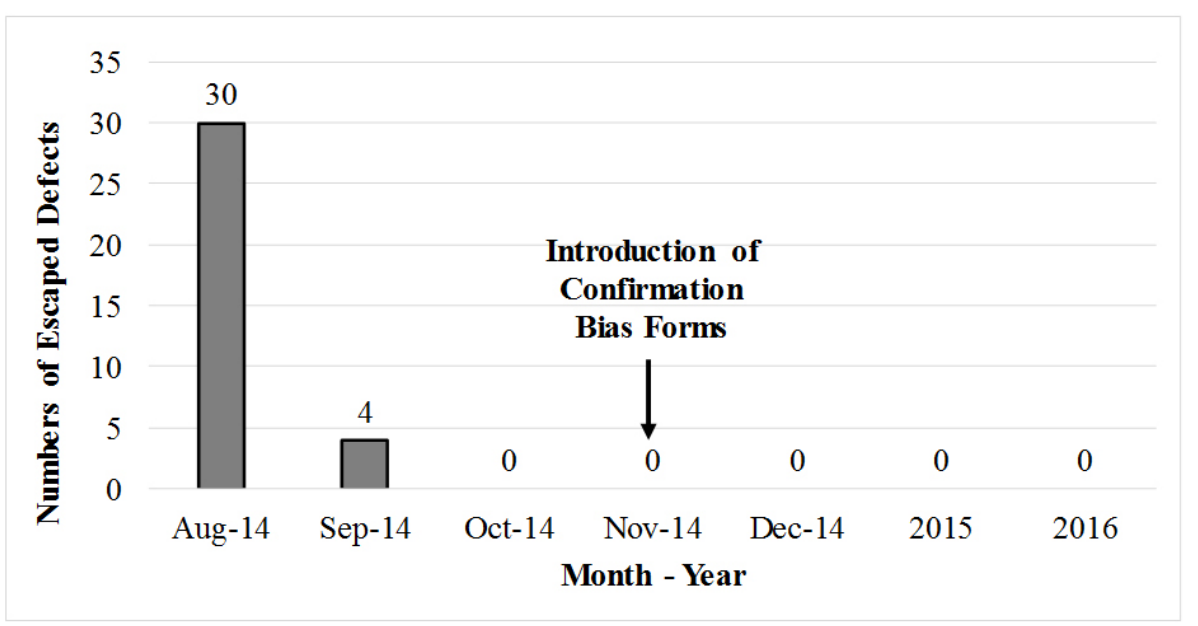

Figure 1. Eliminating confirmation bias in the medication verification process for compounded products Escaped defects were defined as "potential" medications errors that captured prior to leaving the pharmacy and did not reach the patient nor resulted in any harm

\subsection{Process development}

Some studies have shown that in order to reduce or eliminate confirmation bias, it may be prudent to obtain falsifying information on the subject matter to disprove an event rather than prove an event to support one's claim. ${ }^{[9]}$ Therefore, actively seeking and predicting potential medication errors may help disprove the plausibility of an event. Applying this concept, our pharmacy team (comprised of the Executive Director of Pharmacy, Director of Pharmacy Operations, Pharmacy Supervisor and Technician Coordinator) established a new approach to drug verification in late 2013. Through multiple brainstorming and planning sessions, our team constructed an innovative method of verifying medications that minimizes the risk of developing medication errors.

For the non-sterile compounding of oral products and the sterile compounding of IV admixtures, we developed a threestep verification process that does not rely solely on checking against the recipe card for non-sterile oral liquid products, or the product label for sterile IV medications (see Figure 2). Notably, this process integrates a double-check system. The first check occurs when the pharmacist examines images of the original drug vials (or bottles) and syringes (or graduated cylinders) of volume extracted from the active and inactive ingredients. The pharmacist mindfully notes the drug name, dose, and number of capsules or tablets for the product constituents (see Figure 3). Second, the pharmacist predicts the final medication concentration or volume to be prepared without the recipe card or product label. With the recipe card or product label faced down so that the ingredients are not visible, the pharmacist notes the total dose with units, diluents and volumes used, and total volume along with concentration of the final medication product (see Figure 4). After visualizing the process of compounding the product and predicting the final oral or IV product, the second check occurs when the pharmacist verifies against the recipe card or product label and check the expiration date before s/he approves the final product (see Figure 5).

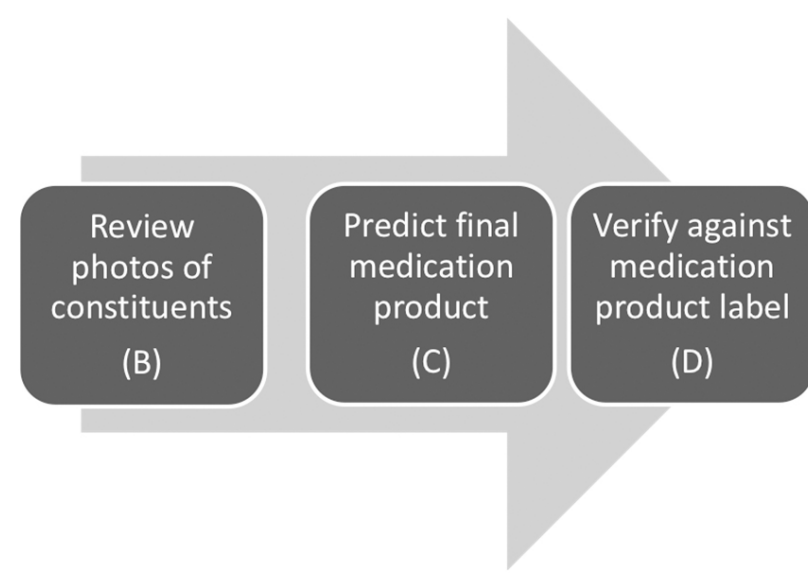

Figure 2. Steps in the verification process for intravenous medication admixtures

This unique double-check method of drug verification (where the pharmacist first checks the drug with the final product label down, and then turns the label around for a second check) 
eliminates the confirmation bias that would have emerged from knowing beforehand what to expect in preparing the medication. The need to predict the medication based on constituents requires a conscious effort, with application of quantitative skills to ensure accurate preparation of the final medication product. For the non-sterile compounding of oral products, we also integrated the use of technology, including iPads and PharmacyKeeper, an online checking system of
MedKeeper (Englewood, CO). PharmacyKeeper is a weband mobile-based product that supports, tracks, and reports (in real-time) pharmacy operations, including the drug verification workflow. The pharmacy technicians photographed the constituents and final product preparations on the iPad and uploaded onto PharmacyKeeper for the pharmacist to check.
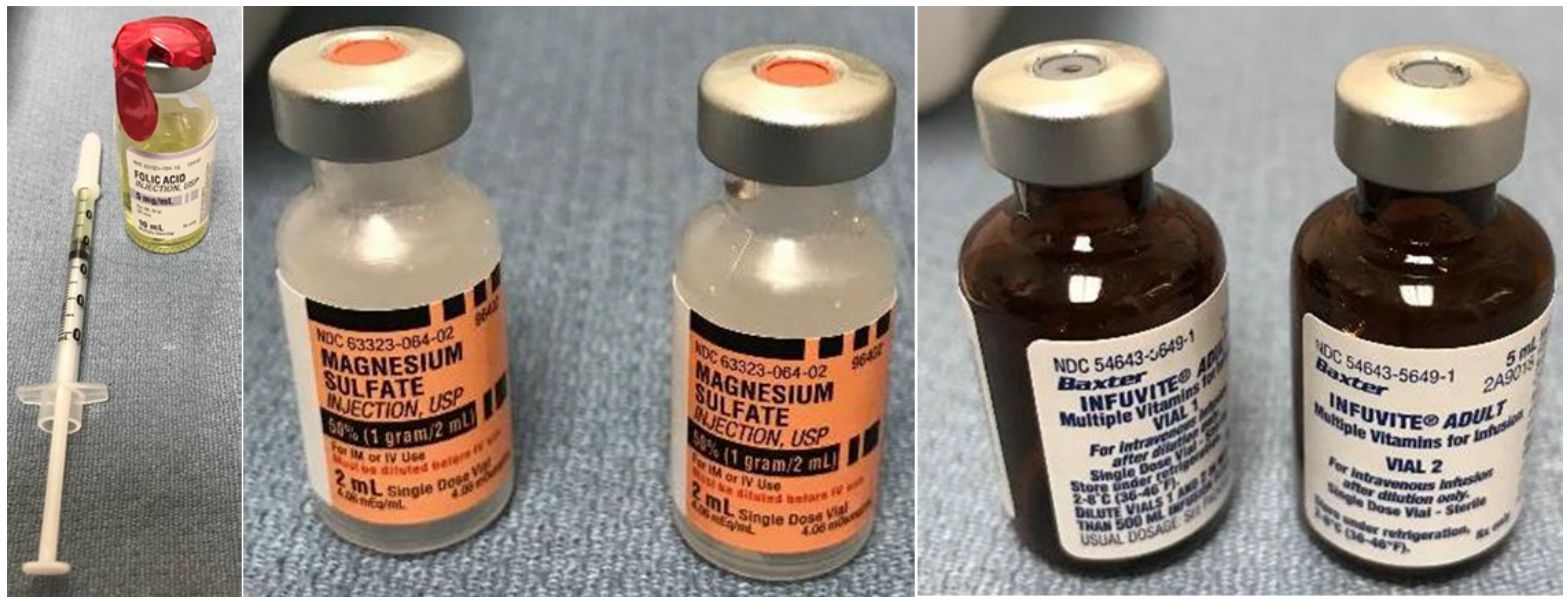

Figure 3. Images of the constituents for intravenous medication admixtures

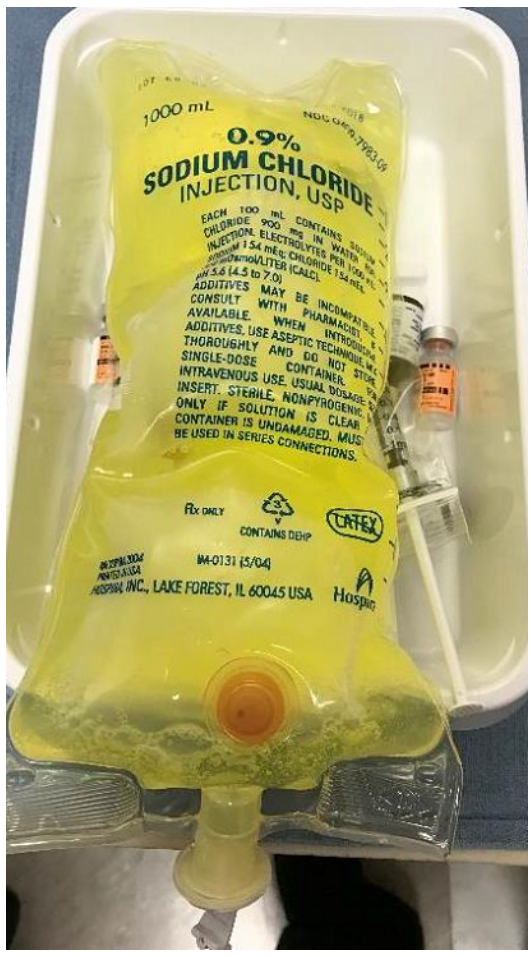

Figure 4. First check without product label for intravenous medication admixtures

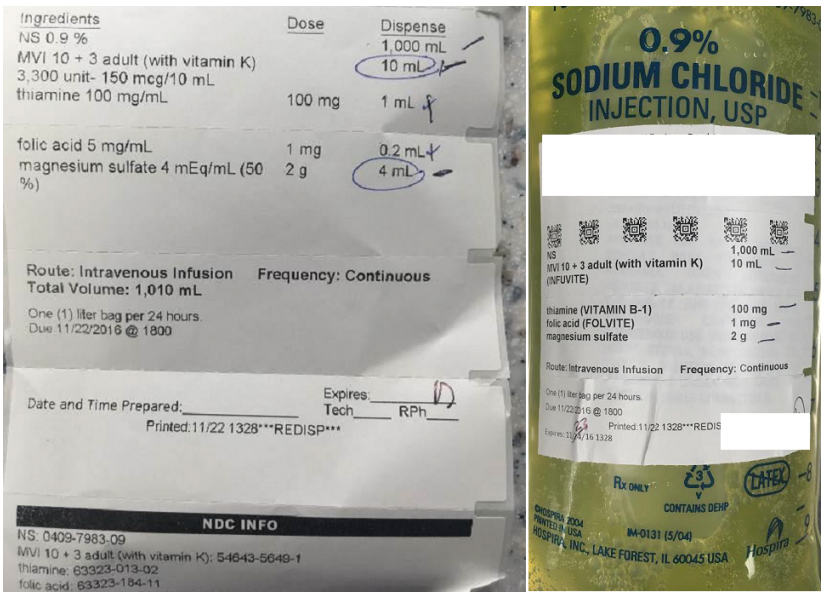

Figure 5. Double check against product label for intravenous medication admixtures

\subsection{Process implementation}

A pilot of our unique verification process was tested at our pharmacy and therapeutics lab that serviced LBMC and MCWHLB for non-sterile oral liquid dilutions in September 2014. The process implementation produced an immediate effect, with only four medication errors reported in September 2014, which was an all-time low (see Figure 1). The effect was most evident when the pharmacist adhered to the 
new process integrated into the workflow. To combat any drift in the designed workflow, our team created a confirmation bias form that required the pharmacist to answer key questions (see Figure 1). The system designed forced the checking pharmacist to follow a certain algorithm to check a specific non-sterile dilution. The fact that this was a requirement to check non-sterile oral medications in the designed workflow forced pharmacist to look at the all the required elements of the compound prior to signing off without the possibility of drift. Using this form for non-sterile oral medications, the pharmacist were required to: (1) record the drug name and strength, (2) calculate the total strength of active ingredients, (3) record diluents and volumes, (4) calculate the concentration expected of the final product, and (5) record whether or not the final product and its concentration matched the recipe card. Completion of this form was required prior to checking the recipe, hence eliminating the expectation factor associated with confirmation bias. Since implementation of our verification process, no escaped defect has been reported. This demonstrates that our confirmation bias-minimization process may be an effective approach to improve patient safety. Furthermore, while the implementation of this process improved the rate of system catches, there was no change in pharmacy staff requirement, indicating its cost neutrality.

\section{Future direction}

As pharmacists, we adopted an oath to apply our knowledge, experience and skills to ensure optimal outcomes for our patients. The art of safe distribution of medications may be overshadowed as the profession of pharmacy continues to expand its clinical enterprise. While clinical pharmacy promotes the effective use of medications, efforts to uncover new approaches to ensure the safe use of medications should continue. The nature of this dual responsibility of pharmacists may create an environment where multitasking can inherently introduce task interference, hence leading to medication errors. Developing and standardizing our unique process of drug verification that involves a double check system and minimization of confirmation bias was successful in reducing potential medication errors. Our approach proved to be safe and efficient, with no additional staffing requirement. In the future, we plan to implement this process in our pharmacy's IV production process that incorporates first doses and hazardous drug compounding. We also plan to educate other departments of our approach with the goal of integrating the double check system and limiting confirmation bias in the mediation use process throughout the hospitals (i.e., chemotherapeutic agents in nursing where the nurses will check the IV bag first to see what they have and then double check with our electronic medical record). Lastly, we plan to conduct a study to evaluate the impact of our approach on medication errors that reached patients, including NCC-MERP Categories C to I, and on patient outcomes, including length of stay, admission to the intensive care unit and all-cause mortality. ${ }^{[13]}$

\section{Conclusion}

Confirmation bias, that results primarily from expectation, is present in healthcare and it may introduce potential medication errors. The role of pharmacists is pivotal in supporting the health of patients through the safe use of medications. As described in this report, the elimination of confirmation bias and incorporation of a double-check system during the medication verification process may significantly reduce escaped defects, notably with cost neutrality in pharmacy staffing. The implementation of this new approach occurred in the pharmacy department and may be integrated hospital wide.

\section{KEY POINTS}

(1) Confirmation bias may occur during the verification step in the medication use process, which may potentially lead to medications errors and consequently, adverse drug events.

(2) The innovative verification process led to no reported errors, demonstrating that our confirmation biasminimization process may be an effective approach to improve patient safety.

(3) All while the implementation of this process improved the rate of system catches, there was no change in pharmacy staff requirement, indicating its cost neutrality.

\section{ACKNOWLEDGEMENTS}

This study was not supported by any funding agency. The authors are solely responsible for the study design, data collection and analysis, decision to publish, or preparation of the manuscript.

We would like to send our gratitude to Willis Shu, PharmD and the pharmacy department administrators and staff at Long Beach Memorial and Millers Children's and Women's Hospital of Long Beach for their support in planning and successfully implementing this innovative process.

\section{Conflicts of InTEREST Disclosure}

The authors declare no conflicts of interest. 


\section{REFERENCES}

[1] Fortescue EB, Kaushal R, Landrigan CP, et al. Prioritizing strategies for preventing medication errors and adverse drug events in pediatric inpatients. Pediatrics. 2003; 111(4 Pt 1): 722-9. PMid:12671103. https://doi.org/10.1542/peds.111.4.722

[2] Smyth RM, Gargon E, Kirkham J, et al. Adverse drug reactions in children-a systematic review. PLoS One. 2012; 7(3): e24061. PMid:22403604. https://doi.org/10.1371/journal.pone.0 024061

[3] Le J, Nguyen T, Law AV, et al. Adverse drug reactions among children over a 10-year period. Pediatrics. 2006; 118(2): 555-62. PMid:16882807. https ://doi .org/10.1542/peds. 2005-2429

[4] Kaushal R, Bates DW, Landrigan C, et al. Medication errors and adverse drug events in pediatric inpatients. JAMA. 2001; 285(16): 2114-20. PMid:11311101. https://doi.org/10.1001/jama. 2 85.16 .2114

[5] Kuo GM, Touchette DR, Marinac JS. Drug errors and related interventions reported by united states clinical pharmacists: The american college of clinical pharmacy practice-based research network medication error detection, amelioration and prevention study. Pharmacotherapy. 2013; 33(3): 253-65. PMid:23378169. https: //doi.org/10.1002/phar.1195

[6] Leonard MS. Patient safety and quality improvement: Medical errors and adverse events. Pediatr Rev. 2010; 31(4): 151-8. PMid:20360409. https://doi.org/10.1542/pir.31-4-151
[7] Institute of Medicine, Committee on Quality of Health Care in America. To err is human: Building a safer health system. National Academies Press; 2000.

[8] Garner SS, Cox TH, Hill EG, et al. Prospective, controlled study of an intervention to reduce errors in neonatal antibiotic orders. $\mathrm{J}$ Perinatol. 2015; 35(8): 631-5. PMid:25836318. https://doi.or g/10.1038/jp.2015.20

[9] Maaskant JM, Vermeulen H, Apampa B, et al. Interventions for reducing medication errors in children in hospital. Cochrane Database Syst Rev. 2015; (3): CD006208. PMid:25756542. https : //doi .or g/10.1002/14651858. CD006208.pub3

[10] Institute for Safe Medication Practices. Illustrating the application of the key elements through the medication flow process. Available from: https: //www.ismp.org/communityRx/aroc/files/Fl ow_Process.pdf (accessed 2017 July 4).

[11] Inattentional blindness: What captures your attention? Institute for Safe Medication Practices Medication Safety Alert. 2012; 18(4) https ://doi .org/10.1016/S1042-0991(15)31897-1

[12] Molloy L, Snyder AH, Srivastava R, et al. Ceftaroline fosamil for methicillin-resistant staphylococcus aureus pulmonary exacerbation in a pediatric cystic fibrosis patient. J Pediatr Pharmacol Ther. 2014; 19(2): 135-40. PMid:25024675. https://doi.org/10.5863/15 51-6776-19.2.135

[13] National Coordinating Council for Medication Error Reporting and Prevention. Available from: http://www.nccmerp.org/types-m edication-errors (Accessed 2017 April 5). 